\title{
A Survey of Software-Defined Networking: Past, Present, and Future of Programmable Networks
}

\author{
Marc Mendonca, Bruno Astuto A. Nunes, Xuan-Nam Nguyen, Katia Obraczka, and Thierry Turletti
}

\begin{abstract}
The idea of programmable networks has recently regained considerable momentum due to the emergence of the Software-Defined Networking (SDN) paradigm. SDN, often referred to as a "radical new idea in networking", promises to dramatically simplify network management and enable innovation through network programmability. This paper surveys the stateof-the-art in programmable networks with an emphasis on SDN. We provide a historic perspective of programmable networks from early ideas to recent developments. Then we present the SDN architecture and the OpenFlow standard in particular, discuss current alternatives for implementation and testing SDNbased protocols and services, examine current and future SDN applications, and explore promising research directions based on the SDN paradigm.
\end{abstract}

Index Terms-Software-Defined Networking, programmable networks, survey, data plane, control plane, virtualization.

\section{INTRODUCTION}

C OMPUTER networks are typically built from a large number of network devices such as routers, switches and numerous types of middleboxes with many complex protocols implemented on them. Network operators are responsible for configuring policies to respond to a wide range of network events and applications. They have to manually transform these high level-policies into low-level configuration commands while adapting to changing network conditions. And often they need to accomplish these very complex tasks with access to very limited tools. As a result, network management and performance tuning is quite challenging and thus errorprone. The fact that network devices are usually verticallyintegrated black boxes exacerbates the challenge network operators and administrators face.

Another almost unsurmountable challenge network practitioners and researchers face has been referred to as "Internet ossification". Because of its huge deployment base and the fact it is considered part of our society's critical infrastructure (just like transportation and power grids), the Internet has become extremely difficult to evolve both in terms of its physical infrastructure as well as its protocols and performance. However, as current and emerging Internet applications and services become increasingly more complex and demanding, it is imperative that the Internet be able to evolve to address these new challenges.

The idea of "programmable networks" has been proposed as a way to facilitate network evolution. In particular, Software

Bruno Astuto A. Nunes, Xuan-Nam Nguyen and Thierry Turletti are with INRIA, France, \{bruno.astuto-arouche-nunes, xuan-nam.nguyen, thierry.turletti\}@inria.fr

Marc Mendonca and Katia Obraczka are with UC Santa Cruz, \{msm, katia\}@soe.ucsc.edu
Defined Networking (SDN) is a new networking paradigm in which the forwarding hardware is decoupled from control decisions. It promises to dramatically simplify network management and enable innovation and evolution. The main idea is to allow software developers to rely on network resources in the same easy manner as they do on storage and computing resources. In SDN, the network intelligence is logically centralized in software-based controllers (at the control plane), and network devices become simple packet forwarding devices (the data plane) that can be programmed via an open interface (e.g., ForCES [40], OpenFlow [71], etc).

SDN is currently attracting significant attention from both academia and industry. A group of network operators, service providers, and vendors have recently created the Open Network Foundation [13], an industrial-driven organization, to promote SDN and standardize the OpenFlow protocol [71]. On the academic side, the OpenFlow Network Research Center [14] has been created with a focus on SDN research. There have also been standardization efforts on SDN at the IETF and IRTF.

The field of software defined networking is quite recent, yet growing at a very fast pace. Still, there are important research challenges to be addressed. In this paper, we survey the stateof-the-art in programmable networks by providing a historic perspective of the field and also describing in detail the SDN paradigm and architecture. The paper is organized as follows: in Section II, it begins by describing early efforts focusing on programmable networks. Section III provides an overview of SDN and its architecture. It also describes the OpenFlow protocol. In Section V, we discuss several SDN applications in areas such as data centers and wireless networking. Section IV describes existing platforms for developing and testing SDN solutions including emulation and simulation tools, SDN controller implementations, as well as verification and debugging tools. Finally, Section VI discusses future research directions related to SDN.

\section{Early Programmable Networks}

SDN has great potential to change the way networks operate, and OpenFlow in particular has been touted as a "radical new idea in networking" [67]. The proposed benefits range from centralized control, simplified algorithms, commoditizing network hardware, eliminating middleboxes, to enabling the design and deployment of third-party 'apps'.

While OpenFlow has received a considerable amount of industry attention, it is worth noting that the idea of programmable networks and decoupled control logic has been 
around for many years. In this section, we provide an overview of early programmable networking efforts, precursors to the current SDN paradigm that laid the foundation for many of the ideas we are seeing today.

a) Open Signaling: The Open Signaling (OPENSIG) working group began in 1995 with a series of workshops dedicated to "making ATM, Internet and mobile networks more open, extensible, and programmable" [32]. They believed that a separation between the communication hardware and control software was necessary but challenging to realize; this is mainly due to vertically integrated switches and routers, whose closed nature made the rapid deployment of new network services and environments impossible. The core of their proposal was to provide access to the network hardware via open, programmable network interfaces; this would allow the deployment of new services through a distributed programming environment.

Motivated by these ideas, an IETF working group was created, which led to the General Switch Management Protocol (GSMP) [39], a general purpose protocol to control a label switch. GSMP allows a controller to establish and release connections across the switch, add and delete leaves on a multicast connection, manage switch ports, request configuration information, request and delete reservation of switch resources, and request statistics." The working group is officially concluded and the latest standards proposal, GSMPv3, was published in June 2002.

b) Active Networking: Also in the mid 1990s, the Active Networking [95], [96] initiative proposed the idea of a network infrastructure that would be programmable for customized services. There were two main approaches being considered, namely: (1) user-programmable switches, with inband data transfer and out-of-band management channels; and (2) capsules, which were program fragments that could be carried in user messages; program fragments would then be interpreted and executed by routers. Despite considerable activity it motivated, Active Networking never gathered critical mass and transferred to widespread use and industry deployment, mainly due to practical security and performance concerns [76].

c) DCAN: Another initiative that took place in the mid 1990s is the Devolved Control of ATM Networks (DCAN) [4]. The aim of this project was to design and develop the necessary infrastructure for scalable control and management of ATM networks. The premise is that control and management functions of the many devices (ATM switches in the case of DCAN) should be decoupled from the devices themselves and delegated to external entities dedicated to that purpose, which is basically the concept behind SDNs. DCAN assumes a minimalist protocol between the manager and the network, in the lines of what happens today in proposals such as OpenFlow. More on the DCAN project can be found at [73].

Still in the lines of SDNs and the proposed decoupling of control and data plane over ATM networks, amongst others, in the work proposed in [99] multiple heterogeneous control architectures are allowed to run simultaneously over single physical ATM network by partitioning the resources of that switch between those controllers. d) 4D Project: Starting in 2004, the 4D project [87], [48], [29] advocated a clean slate design that emphasized separation between the routing decision logic and the protocols governing the interaction between network elements. It proposed giving the "decision" plane a global view of the network, serviced by a "dissemination" and "discovery" plane, for control of a "data" plane for forwarding traffic. These ideas provided direct inspiration for later works such as NOX [49], which proposed an "operating system for networks" in the context of an OpenFlow-enabled network.

e) NETCONF: In 2006, the IETF Network Configuration Working Group proposed NETCONF [42] as a management protocol for modifying the configuration of network devices. The protocol allowed network devices to expose an API through which extensible configuration data could be sent and retrieved. Although the protocol accomplishes the goal of simplifying device (re)configuration and acts as a building block, there is no separation of the data and control plane. A network with NETCONF should not be regarded as fully programmable as any new functionality would have to be implemented at both the network device and the manager for management to take place; furthermore, it is designed primarily to aid automated configuration and not for enabling direct control of state data. Nevertheless, it is a useful management tool that may be used in parallel on hybrid switches supporting other solutions that enable programmable networking.

The working group is currently active and the latest proposed standard was published in June 2011.

f) Ethane: The immediate predecessor to OpenFlow was the SANE / Ethane project [34], which, in 2006, defined a new network architecture for enterprise networks. Ethane's focus was on using a centralized controller to manage policy and security in a network. A notable example is providing identitybased access control. Similarly to SDN, Ethane employed two components: a controller to decide if a packet should be forwarded, and an Ethane switch consisting of a flow table and a secure channel to the controller.

Ethane laid the foundation for what would become Software-Defined Networking. To put Ethane in the context of today's SDN paradigm, Ethane's identity-based access control would likely be implemented as an application on top of a SDN controller such as NOX [49], Maestro [30], Beacon [1], SNAC [20], Helios [6], etc.

\section{SOFTWARE-Defined NETWORKING ARCHITECTURE}

Data communication networks typically consist of enduser devices, or hosts interconnected by the network infrastructure. This infrastructure is shared by hosts and employs switching elements such as routers and switches as well as communication links to carry data between hosts. Routers and switches are usually "closed" systems, often with limitedand vendor-specific control interfaces. Therefore, once deployed and in production, it is quite difficult for current network infrastructure to evolve; in other words, deploying new versions of existing protocols (e.g., IPv6), not to mention deploying completely new protocols and services is an almost 


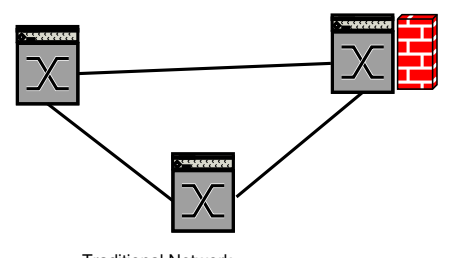

Traditional Network (with distributed control and middleboxes)

Fig. 1. The SDN architecture decouples control logic from the forwarding hardware, and enables the consolidation of middleboxes, simpler policy management, and new functionalities.

insurmountable obstacle in current networks. The Internet, being a network of networks, is no exception.

As mentioned previously, the so-called Internet "ossification" [71] is largely attributed to the tight coupling between the data- and control planes which means that decisions about data flowing through the network are made on-board each network element. In this type of environment, the deployment of new network applications or functionality is decidedly nontrivial, as they would need to be implemented directly into the infrastructure. Alternatively, workarounds such as using "middleboxes" (e.g., firewalls, Intrusion Detection Systems, Network Address Translators, etc.) overlayed atop the underlying network infrastructure have been proposed and deployed as a way to circumvent the network ossification effect. Content Delivery Networks (CDNs) [82] are a good example. Furthermore, even straightforward tasks such as configuration or policy enforcement may require a good amount of effort due to the lack of a common control interface to the various network devices.

Software-Defined Networking was developed to facilitate innovation and enable simple programmatic control of the network data-path. As visualized in Figure 1, the separation of the forwarding hardware from the control logic allows easier deployment of new protocols and applications, straightforward network visualization and management, and consolidation of various middleboxes into software control. Instead of enforcing policies and running protocols on a convolution of scattered devices, the network is reduced to "simple" forwarding hardware and the decision-making network controller(s). The forwarding hardware consists of (1) a flow table containing an entry and an action to take on active flows; and (2) an abstraction layer that securely communicates with a controller about new entries that are not currently on the flow table.

\section{A. Current SDN Architectures}

In this section, we review two well-known SDN systems, namely ForCES [40] and Openflow [71]. Both OpenFlow and ForCES follow the basic SDN principle of separation between control- and data-planes; and both standardize information exchange between planes. However, they are technically very different in terms of design, architecture, forwarding model, and protocol interface. g) ForCES: According to the approach proposed by the IETF ForCES (Forwarding and Control Element Seperaration) Working Group [40], the network device's internal architecture is redefined having the control element separated from the forwarding element. However, the network device is still represented as a single entity. The driving use case provided by the working group considers the desire to combine new forwarding hardware with third-party control within a single network device. Thus, the control- and data planes are kept within close proximity (e.g., same box or room). In contrast, the control plane is ripped entirely from the network device in "OpenFlow-like" SDN systems.

ForCES defines a logic entity called the Forwarding Element (FE), that implements the ForCES protocol, i.e. the communication protocol between FEs and CEs, and is responsible for using the underlying hardware to provide per-packet handling. Another logic entity defined in ForCES is the Control Element (CE), that executes control and signaling functions and employs the ForCES protocol to instruct FEs on how to handle packets. The protocol works based on a master-slave model, where FEs are slaves and CEs are masters.

An important building block of the ForCES architecture is the LFB (Logical Function Block). The LFB is a well-defined functional block residing on the FEs that is controlled by CEs via the ForCES protocol. The LFB enables the CEs to control the FEs' configuration; the LFB may reside on the FE's data plane and is used to process packets.

ForCES has been undergoing standardization since 2003, and the working group has published a variety of documents including an applicability statement, an architectural framework defining the entities and their interactions, a modeling language defining the logical functions within a forwarding element, and a protocol for communication between the control and forwarding elements within a network element. The working group is currently active.

h) OpenFlow: Driven by the SDN principle of decoupling the control- and data forwarding planes, OpenFlow [71], like ForCES, standardizes information exchange between the two planes.

In the OpenFlow architecture, the forwarding device, or OpenFlow switch, contains one or more flow tables. Flow tables consist of flow entries, each of which determines how packets belonging to a flow will be processed and forwarded. Flow entries typically consist of: (1) match fields used to match incoming packets; match fields may contain information found in the packet header, ingress port, and metadata; (2) counters, used to collect statistics for the particular flow, such as number of received packets, number of bytes and duration of the flow; and (3) a set of instructions, or actions, to be applied upon a match; they dictate how to handle matching packets.

Upon a packet arrival at the OpenFlow switch, packet header fields are extracted and matched against the matching fields portion of the flow table entries. This matching starts at the first flow table and may continue through subsequent flow tables. If a matching entry is found, the switch applies the appropriate set of instructions associated with the matched flow entry. If the flow table look-up procedure does not result on a match, the action taken by the switch will depend on the instructions 
defined by the table-miss flow entry. Every flow table must contain a table-miss entry in order to handle table misses. This particular entry specifies a set of actions to be performed when no match is found for an incoming packet. These actions include dropping the packet, continue the matching process on the next flow table, or forward the packet to the controller over the OpenFlow channel. Another possibility, in the case of hybrid switches, i.e., switches that have both OpenFlowand non-OpenFlow ports, is to forward non-matching packets using regular IP forwarding.

The communication between controller and switch happens via OpenFlow protocol, which defines a set of messages that can be exchanged between these entities over a secure channel. Using the OpenFlow protocol a remote controller can, for example, add, update, or delete flow entries from the switch's flow tables. That can happen reactively (in response to a packet arrival) or proactively.

1) Discussion: In [106], the similarities and differences between ForCES and OpenFlow are discussed. Among the differences, they highlight the fact that the forwarding model used by ForCES relies on the Logical Function Blocks (LFBs), while OpenFlow uses flow tables. Actions associated with a flow can be combined to provide greater control and flexibility for the purposes of network management, administration, and development. In ForCES the combination of different LFBs can also be used to achieve the same goal.

We should also re-iterate that ForCES does not follow the same SDN model underpinning OpenFlow, but can be used to achieve the same goals and implement similar functionality [106].

The strong support from industry, research, and academia that the Open Networking Foundation (ONF) and its SDN proposal, OpenFlow, has been able to gather is quite impressive. The resulting critical mass from these different sectors has produced a significant number of deliverables in the form of research papers, reference software implementations, and even hardware. So much so that some argue that OpenFlow's SDN architecture is the current SDN de-facto standard. In line with this trend, the remainder of this section focuses on OpenFlow's SDN model. More specifically, we will describe the different components of the SDN architecture, namely: the switch, the controller, and the interfaces present on the controller for communication with forwarding devices (southbound communication) and network applications (northbound communication). Section IV also has an OpenFlow focus as it describes existing platforms for SDN development and testing, including emulation and simulation tools, SDN controller implementations, as well as verification and debugging tools. Our discussion of future SDN applications and research directions is more general and is SDN architecture agnostic.

\section{B. Forwarding Devices}

Forwarding devices such as switches and routers in a software-defined network are often represented as basic forwarding hardware accessible via an open interface, as the control logic and algorithms are off-loaded to a controller.

In an OpenFlow network, switches come in two varieties: pure and hybrid. Pure OpenFlow switches have no legacy features or on-board control, and completely rely on a controller for forwarding decisions. Hybrid switches support OpenFlow in addition to traditional operation and protocols. Most commercial switches available today are hybrids.

Though at first glance this would appear to simplify the switching hardware, flow-based SDN architectures such as OpenFlow may utilize additional forwarding table entries, buffer space, and statistical counters that are difficult to implement in traditional ASIC switches. Some recent proposals [68], [74] have advocated adding a general-purpose CPU, either onswitch or nearby, that may be used to supplement or take over certain functions and reduce the complexity of the ASIC design. This would have the added benefit of allowing greater flexibility for on-switch processing as some aspects would be software-defined.

In some earlier work [69], authors apply network processor based acceleration cards to perform OpenFlow switching. They propose and describe the design options and report results that show a $20 \%$ reduction on packet delay. Also, in [94], an architectural design to improve look-up performance of OpenFlow switching in Linux is proposed. Preliminary results reported show a packet switching throughput increase up to $25 \%$ compared to the throughput of regular software-based OpenFlow switching. Another study on dataplane performance over Linux based Openflow switching is presented in [25]. In the paper, authors compare OpenFlow switching, layer2 Ethernet switching and layer-3 IP routing performance. Fairness, forwarding throughput and packet latency in diverse load conditions are analyzed. In [58], authors derive a basic model for the forwarding speed and blocking probability of an OpenFlow switch, where the parameters for the model are drawn from measurements of switching times of current OpenFlow hardware, combined with an OpenFlow controller. Simulation validation of the proposed model is presented.

\section{The Controller}

The decoupled system has been compared to an operating system [49], in which the controller provides a programmatic interface to the network, where applications can be written to perform management tasks and offer new functionalities. A layered view of this model is illustrated in Figure 2. This view assumes the control is centralized and applications are written as if the network is a single system. While this simplifies policy enforcement and management tasks, the bindings must be closely maintained between the control and the network forwarding elements.

1) Control models: An initial concern that arises when offloading control from the switching hardware is the scalability and performance of the network controller(s). The original Ethane [34] controller, hosted on a commodity desktop machine, was tested to handle up to 11,000 new flow requests per second and responded within 1.5 milliseconds. A more recent study [98] of several OpenFlow controller implementations (NOX-MT, Maestro, Beacon), conducted on a larger emulated network with 100,000 endpoints and up to 256 switches, found that all were able to handle at least 50,000 new flow requests per second in each of the tested 


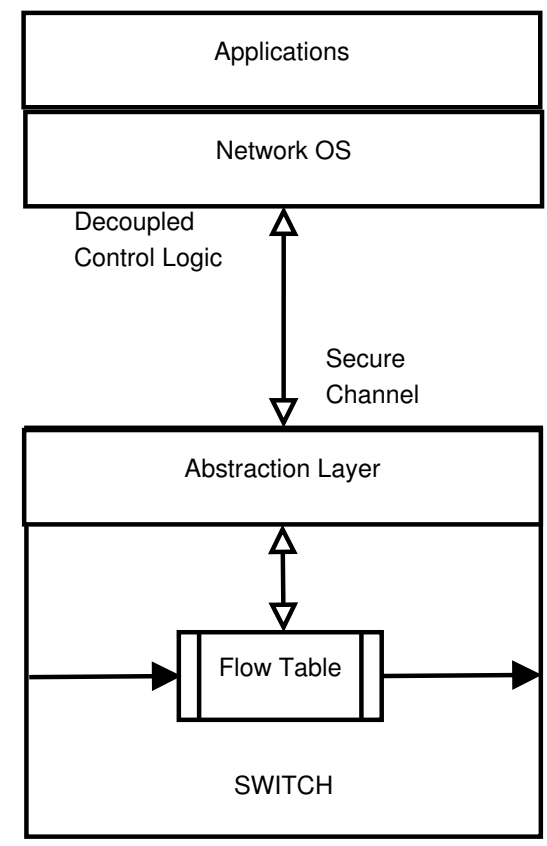

Fig. 2. The separated control logic can be viewed as a network operating system, upon which applications can be built to "program" the network.

scenarios. On an eight-core machine, the multi-threaded NOXMT implementation handled 1.6 million new flow requests per second with an average response time of 2 milliseconds. As the results show, a single controller is able to handle a surprising number of new flow requests, and should be able to manage all but the largest networks. Furthermore, new controllers under development such as McNettle [103] target powerful multicore servers and are being designed to scale up to large data center workloads ( 20 million flows requests per second and up to 5000 switches). Nonetheless, multiple controllers may be used to reduce latency or increase fault tolerance.

A related concern is the controller placement problem [54], which attempts to determine both the optimal number of controllers and their location within the network topology, often choosing between optimizing for average and worst case latency. Also, control modeling impacts greatly the network scalability. Authors in [110] go over some important scalability issues and discuss some scalability trade-offs in softwaredefined networks design.

In the following, we go over some of these SDN design options and discuss different methods of controlling a softwaredefined network, many of which are interrelated:

- Centralized vs. Distributed

Although protocols such as OpenFlow specify that a switch is controlled by a controller and therefore appears to imply centralization, software-defined networks may have either a centralized or distributed controlplane. Though controller-to-controller communication is not defined by OpenFlow, it is necessary for any type of distribution or redundancy in the control-plane.

A physically centralized controller represents a single point of failure for the entire network; therefore, OpenFlow allows the connection of multiple controllers to a switch, which would allow backup controllers to take over in the event of a failure.

Onix [63] and HyperFlow [97] take the idea further by attempting to maintain a logically centralized but physically distributed control plane. This decreases the look-up overhead by enabling communication with local controllers, while still allowing applications to be written with a simplified central view of the network. The potential downside are trade-offs [65] that may be made with consistency and staleness when distributing state throughout the control plane, which has the potential to cause applications that believe they have an accurate view to act incorrectly.

A hybrid approach, such as Kandoo [52], can utilize local controllers for local applications and redirect to a global controller for decisions that require a centralized network state. This reduces the load on the global controller by filtering the number of new flow requests, while also providing the data-path with faster responses for requests that can be handled by a local control application.

A software-defined network can also have some level of logical decentralization, with multiple logical controllers. An interesting type of proxy controller, called Flowvisor [89], can be used to add a level of network virtualization to OpenFlow networks and allow multiple controllers to simultaneously control overlapping sets of physical switches. Initially developed to allow experimental research to be conducted on deployed networks alongside production traffic, it also facilitates and demonstrates the ease of deploying new services in SDN environments.

A logically decentralized control plane may also be the result of an inter-network spanning multiple domains of control. Though the domains may not agree to centralized control, a certain level of sharing may be appropriate (e.g., to ensure service level agreements are met for traffic flowing between domains).

- Control Granularity

Traditionally, the basic unit of networking has been the packet. Each packet contains the address information necessary for a network switch to make individual routing decisions. However, most applications send data as a flow of many individual packets. A network that wishes to provide QoS or service guarantees to certain applications may benefit from individual flow-based control. Control can be further abstracted to an aggregated flow-match, rather than individual flows. Flow aggregation may be based on source, destination, application, or any combination thereof.

In a software-defined network with remote control of network hardware, overhead is caused by traffic between the data-plane and control-plane. As such, using packetbased granularity would incur additional delay as the controller would have to make a decision for each arriving packet. When controlling individual flows, the decision made for the first packet of the flow can be applied to all subsequent packets of the flow within the dataplane. The overhead may be further reduced by grouping flows together, such as all traffic between two hosts, and performing control decisions on the aggregated flows. 
- Reactive vs. Proactive Policies

With a reactive control model, such as the one proposed by Ethane [34], the forwarding elements must consult a controller each time a decision must be made, such as when a new packet flow reaches a switch. In the case of flow-based control granularity, there will be a small performance delay as the first packet of each new flow is forwarded to the controller for decision (e.g., forward or drop), after which future traffic packets within that flow will travel at line rate within the forwarding hardware. While the first-packet delay is negligible in many cases, it may be a concern if the controller is geographically remote (though this can be mitigated by physically distributing the controller [97]) or if most flows are short-lived, such as single-packet flows. There are also some scalability issues in larger networks, as the controller throughput must be able to handle the volume of new flow requests.

An alternative proactive approach is to push policy rules out from the controller to the switches. A good example of this approach is DIFANE [112], which partitions rules over a hierarchy of switches, such that the controller rarely needs to be consulted about new flows and traffic is kept within the data-plane. In their experiments, DIFANE reduces first-packet delay from a $10 \mathrm{~ms}$ average RTT with a centralized NOX controller to a $0.4 \mathrm{~ms}$ average RTT with the DIFANE model for new single-packet flows. It also may greatly increase the new flow throughput, as the tested version of NOX achieved a peak of 50,000 singlepacket flows per second while the DIFANE solution achieved 800,000 single-packet flows per second. Interestingly, the authors note the OpenFlow switch's local controller implementation becomes a bottleneck before the central NOX controller, as they learned commercial OpenFlow switch implementations were limited to sending 60-330 new flows requests per second at the time of their publication (2010).

As shown in Figure 3, a controller that strives to act as a network operating system must implement at least two interfaces: a "southbound" interface that allows switches to communicate with the controller and a "northbound" interface that presents a programmable API to network control and highlevel policy applications/services.

\section{Southbound Communication: Controller-Switch}

An important aspect of SDNs is the link between the data-plane and the control-plane. As forwarding elements are controlled by an open interface, it is important that this link remains available and secure.

The OpenFlow protocol can be viewed as one possible implementation of controller-switch interactions, as it defines the communication between the switching hardware and a network controller. For security, OpenFlow 1.3.0 provides optional support for encrypted TLS communication and a certificate exchange between the switches/controller(s); however, the exact implementation and certificate format is not currently specified. Also outside the scope of the current specification

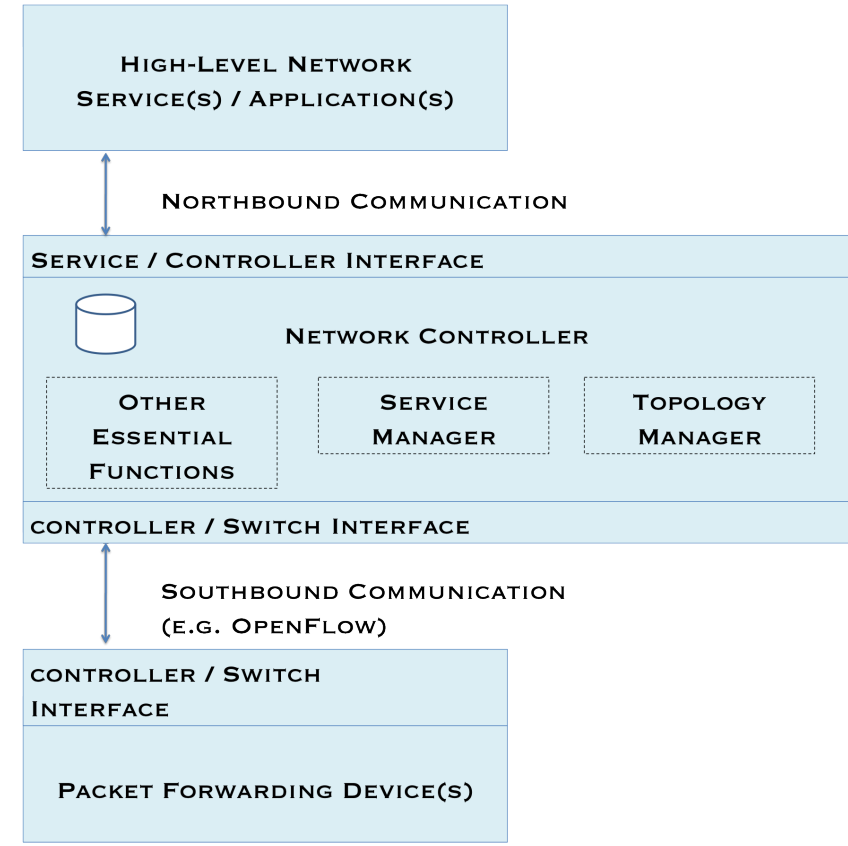

Fig. 3. A controller with a northbound and southbound interface.

are fine-grained security options regarding scenarios with multiple controllers, as there is no method specified to only grant partial access permissions to an authorized controller. We examine OpenFlow controller implementation options in greater detail in Section IV.

\section{E. Northbound Communication: Controller-Service}

External management systems or network services may wish to extract information about the underlying network or control an aspect of network behavior or policy. Additionally, controllers may find it necessary to communicate with each other for a variety of reasons: an internal control app may need to reserve resources across multiple domains of control, a primary controller may need to share policy information with a backup, etc.

Unlike controller-switch communication, there is no currently accepted standard for northbound interactions and they are more likely to be implemented on an ad hoc basis for particular applications. We discuss this further in Section VI.

\section{SDN DEVELOPMENT TOOLS}

SDN facilitates network evolution and innovation by allowing rapid deployment of new services and protocols. In this section, we provide an overview of currently available tools and environments for developing SDN-based services and protocols.

\section{A. Emulation and Simulation Tools}

Mininet [64] allows an entire OpenFlow network to be emulated on a single machine, simplifying the initial development and deployment process. New programs can first be developed and tested on an emulation of the anticipated deployment network before moving to the actual hardware. By default 
Mininet supports OpenFlow v1.0, though it may be modified to support a software switch that implements a newer release.

The ns-3 [55] network simulator supports OpenFlow switches within its environment, though the current version only implements OpenFlow v0.89.

\section{B. Available Software Switch Platforms}

There are currently several SDN software switches available that can be used, for example, to run a SDN testbed or when developing services over SDN. Table I presents a list of current software switch implementations with a brief description including implementation language and the OpenFlow standard version that the current implementation supports.

\section{Native SDN Switches}

One of the main SDN enabling technologies currently being implemented in commodity networking hardware is the OpenFlow standard. In this section we do not intend to give a complete detailed overview of OpenFlow enabled hardware and makers, but rather give a brief list of a few options on what is currently available in the market and give a status about OpenFlow version compliance. One clear evidence of industry's strong commitment to SDN is the availability of commodity network hardware that are OpenFlow enabled. Table II lists commercial switches that are currently available, their manufacturer, and the version of OpenFlow they implement.

\begin{tabular}{|l|c|c|}
\hline Maker & Switch Model & Version \\
\hline Hewlett-Packard & $\begin{array}{r}\text { 8200zl, 6600, 6200zl, } \\
\text { 5400zl, and 3500/3500yl }\end{array}$ & $\mathrm{v} 1.0$ \\
\hline Brocade & NetIron CES 2000 Series & $\mathrm{v} 1.0$ \\
\hline IBM & RackSwitch G8264 & $\mathrm{v} 1.0$ \\
\hline NEC & PF5240 PF5820 & $\mathrm{v} 1.0$ \\
\hline Pronto & 3290 and 3780 & $\mathrm{v} 1.0$ \\
\hline Juniper & Junos MX-Series & $\mathrm{v} 1.0$ \\
\hline Pica8 & P-3290, P-3295, P-3780 and P-3920 & $\mathrm{v} 1.2$ \\
\hline
\end{tabular}

TABLE II

MAIN CURRENT AVAILABLE COMMODITY SWITCHES BY MAKERS, COMPLIANT WITH THE OPENFLOW STANDARD.

\section{Available Controller Platforms}

Table III shows a snapshot of current controller implementations. To date, all the controllers in the table support the OpenFlow protocol version 1.0, unless stated otherwise. Below, we provide a brief overview of the controllers listed in Table III.

- POX [17] is a general, open-source SDN controller written in Python.

- NOX [49] was the first OpenFlow controller written in Python and $\mathrm{C}++$.

- MUL [9] is an OpenFlow controller that has a C-based multi-threaded infrastructure at its core. It supports a multi-level north-bound interface (see Section III-E) for application development.

- Maestro [30] is a network operating system based on Java; it provides interfaces for implementing modular network control applications and for them to access and modify network state.
- Trema is a framework for developing OpenFlow controllers written in Ruby and C.

- Beacon is a cross-platform, modular, Java-based OpenFlow controller that supports event-based and threaded operation.

- Jaxon [8] is a Java-based OpenFlow controller based on NOX.

- Helios [6] is an extensible C-based OpenFlow controller that provides a programmatic shell for performing integrated experiments.

- Floodlight [5] is a Java-based OpenFlow controller, based on the Beacon implementation, that works with physicaland virtual- OpenFlow switches.

- SNAC [20] is an OpenFlow controller based on NOX-0.4, which uses a web-based, user-friendly policy manager to manage the network, configure devices, and monitor events.

- Ryu [18] is an SDN operating system that aims to provide logically centralized control and APIs to create new network management and control applications. Ryu fully supports OpenFlow v1.0, v1.2, v1.3, and the Nicira Extensions.

- NodeFlow [10] is an OpenFlow controller written in JavaScript for Node.JS [11].

- ovs-controller [15] is a simple OpenFlow controller reference implementation with Open vSwitch for managing any number of remote switches through the OpenFlow protocol; as a result the switches function as L2 MAClearning switches or hubs.

Included in Table III are also two special purpose controller implementations: Flowvisor [89], mentioned previously, and RouteFlow [79]. The former acts as a transparent proxy between OpenFlow switches and multiple OpenFlow controllers. It is able to create network slices and can delegate control of each slice to a different controller, also promoting isolation between slices. RouteFlow, on the other hand, is an open source project to provide virtualized IP routing over OpenFlow capable hardware. It is composed by an OpenFlow Controller application, an independent server, and a virtual network environment that reproduces the connectivity of a physical infrastructure and runs IP routing engines. The routing engines generate the forwarding information base (FIB) into the Linux IP tables according to the routing protocols configured (e.g., OSPF, BGP). An extension of RouteFlow is presented in [88], which discusses Routing Control Platforms (RCPs) in the context of OpenFlow/SDN. They propose a controller-centric networking model along a prototype implementation of an autonomous-system-wide abstract BGP routing service.

\section{E. Code Verification and Debugging}

Verification and debugging tools are vital resources for traditional software development and are no less important for SDN. Indeed, for the idea of portable network "apps" to be successful, network behavior must be thoroughly tested and verified.

NICE [33] is an automated testing tool used to help uncover bugs in OpenFlow programs though model checking and symbolic execution. 


\begin{tabular}{|l|c|l|c|}
\hline Software Switch & Implementation & Overview & Version \\
\hline Open vSwitch [15] & C/Python & $\begin{array}{l}\text { Open source software switch that aims to implement a switch platform } \\
\text { in virtualized server environments. Supports standard management } \\
\text { interfaces and enables programmatic extension and control of the } \\
\text { forwarding functions. Can be ported into ASIC switches. }\end{array}$ \\
\hline Pantou/OpenWRT [16] & $\mathrm{C}$ & Turns a commercial wireless router or Access Point into an OpenFlow-enabled switch. & v1.0 \\
\hline ofsoftswitch13 [12] & $\mathrm{C} / \mathrm{C}++$ & OpenFlow 1.3 compatible user-space software switch implementation. & v1.3 \\
\hline Indigo [7] & $\mathrm{C}$ & $\begin{array}{l}\text { Open source OpenFlow implementation that runs on physical switches and uses } \\
\text { the hardware features of Ethernet switch ASICs to run OpenFlow. }\end{array}$ & v1.0 \\
\hline
\end{tabular}

TABLE I

CURRENT SOFTWARE SWITCH IMPLEMENTATIONS COMPLIANT WITH THE OPENFLOW STANDARD.

\begin{tabular}{|l|c|c|c|}
\hline Controller & Implementation & Open Source & Developer \\
\hline POX [17] & Python & Yes & Nicira \\
\hline NOX [49] & Python/C++ & Yes & Nicira \\
\hline MUL [9] & C & Yes & Kulcloud \\
\hline Maestro [30] & Java & Yes & Rice University \\
\hline Trema [21] & Ruby/C & Yes & NEC \\
\hline Beacon [1] & Java & Yes & Stanford \\
\hline Jaxon [8] & Java & Yes & Independent Developers \\
\hline Helios [6] & C & No & NEC \\
\hline Floodlight [5] & Java & Yes & BigSwitch \\
\hline SNAC [20] & C++ & No & Nicira \\
\hline Ryu [18] & Python & Yes & NTT, OSRG group \\
\hline NodeFlow [10] & JavaScript & Yes & Independent Developers \\
\hline ovs-controller [15] & C & Yes & Independent Developers \\
\hline Flowvisor [89] & C & Yes & Stanford/Nicira \\
\hline RouteFlow [79] & C++ & Yes & CPQD \\
\hline
\end{tabular}

TABLE III

CURRENT CONTROLLER IMPLEMENTATIONS COMPLIANT WITH THE OPENFLOW STANDARD.

Anteater [70] takes a different approach by attempting to check network invariants that exist in the data plane, such as connectivity or consistency. The main benefit of this approach is that it is protocol-agnostic; it will also catch errors that result from faulty switch firmware or inconsistencies with the control plane communication. VeriFlow [60] has a similar goal, but goes further by proposing a real-time verification tool that resides between the controller and the forwarding elements. This adds the potential benefit of being able to halt bad rules that will cause anomalous behavior before they reach the network.

Other efforts propose debugging tools that provide insights gleaned from control plane traffic. OFRewind [107] allows network events (control and data) to be recorded at different granularities and later replayed to reproduce a specific scenario, granting the opportunity to localize and troubleshoot the events that caused the network anomaly. $n d b$ [50] implements breakpoints and packet-backtraces for SDN. Just as with the popular software debugger $g d b$, users can pinpoint the events that lead to an error by pausing execution at a breakpoint, or, using a packet backtrace, show the sequence of forwarding actions seen by that packet. STS [19] is a software-defined network troubleshooting simulator. It is written in python and depends on POX. It simulates the devices in a given network allowing for testing cases and identifying the set of inputs that generates a given error.

\section{SDN APPLICATIONS}

Software-defined networking has applications in a wide variety of networked environments. By decoupling the controland data planes, programmable networks enable customized control, an opportunity to eliminate middleboxes, as well as simplified development and deployment of new network services and protocols. Below, we examine different environments for which SDN solutions have been proposed or implemented.

\section{A. Enterprise Networks}

Enterprises often run large networks, while also having strict security and performance requirements. Furthermore, different enterprise environments can have very different requirements, characteristics, and user population, For example, University networks can be considered a special case of enterprise networks: in a University environment, many of the connecting devices are temporary and not controlled by the University, further challenging security and resource allocation. Additionally, Universities must often provide support for research testbeds and experimental protocols.

Adequate management is critically important in Enterprise environments, and SDN can be used to programmatically enforce and adjust network policies as well as help monitor network activity and tune network performance.

Additionally, SDN can be used to simplify the network by ridding it from middleboxes and integrating their functionality within the network controller. Some notable examples of middlebox functionality that has been implemented using SDN include NAT, firewalls, load balancers [51] [104], and network access control [80], etc. In the case of more complex middleboxes with functionalities that cannot be directly implemented without performance degradation (e.g., deep packet inspection), SDN can be used to provide unified control and management[46].

The work presented in [86] addresses the issues related to consistent network updates. Configuration changes are 
a common source of instability in networks and can lead to outages, security flaws, and performance disruptions. In [86], a set of high-level abstractions are proposed that allow network administrators to update the entire network, guaranteeing that every packet traversing the network is processed by exactly one consistent global network configuration. To support these abstractions, several OpenFlow-based update mechanisms were developed.

As discussed in earlier sections, OpenFlow evolved from Ethane [34], a network architecture designed specifically to address the issues faced by enterprise networks.

\section{B. Data Centers}

Data centers have evolved at an amazing pace in recent years, constantly attempting to meet increasingly higher and rapidly changing demand. Careful traffic management and policy enforcement is critical when operating at such large scales, especially when any service disruption or additional delay may lead to massive productivity and/or profit loss. Due to the challenges of engineering networks of this scale and complexity to dynamically adapt to application requirements, it is often the case that data centers are provisioned for peak demand; as a result, they run well below capacity most of the time but are ready to rapidly service higher workloads.

An increasingly important consideration is energy consumption, which has a non-trivial cost in large-scale data centers. Heller et al. [53] indicates that much research has been focused on improved servers and cooling (70\% of total energy) through better hardware or software management, but the data center's network infrastructure (which accounts for $10-20 \%$ of the total energy cost) still consumed 3 billion $\mathrm{kWh}$ in 2006. They propose ElasticTree, a network-wide power manager that utilizes SDN to find the minimum-power network subset which satisfies current traffic conditions and turns off switches that are not needed. As a result, they show energy savings between $25-62 \%$ under varying traffic conditions. One can imagine that these savings can be further increased if used in parallel with server management and virtualization; one possibility is the Honeyguide[90] approach to energy optimization which uses virtual machine migration to increase the number of machines and switches that can be shutdown.

However, not all SDN solutions may be appropriate in high performance networks. While simplified traffic management and visibility are useful, it must be sensibly balanced with scalability and performance overheads. Curtis et al. [37] believe that OpenFlow excessively couples central control and complete visibility, when in reality only "significant" flows need to be managed; this may lead to bottlenecks as the control-data communication adds delay to flow setup while switches are overloaded with thousands of flow table entries. Though aggressive use of proactive policies and wild-card rules may resolve that issue, it may undermine the ability of the controller to have the right granularity to effectively manage traffic and gather statistics. Their framework, DevoFlow, proposes some modest design changes to keep flows in the data plane as much as possible while maintaining enough visibility for effective flow management. This is accomplished by pushing responsibility over most flows back to the switches and adding more efficient statistics collection mechanisms, through which "significant" flows (e.g. long-lived, high-throughput) are identified and managed by the controller. In a load-balancing simulation, their solution had 10-53 times fewer flow table entries and 10-42 times fewer control messages on average over OpenFlow.

\section{Infrastructure-based Wireless Access Networks}

Several efforts have focused on ubiquitous connectivity in the context of infrastructure-based wireless access networks, such as cellular and WiFi.

For example, the OpenRoads project [109], [108] envisions a world in which users could freely and seamlessly move across different wireless infrastructures which may be managed by various providers. They propose the deployment of a SDN-based wireless architecture that is backwardscompatible, yet open and sharable between different service providers. They employ a testbed using OpenFlow-enabled wireless devices such as WiFi APs and WiMAX base stations controlled by NOX- and Flowvisor controllers and show improved performance on handover events. Their vision provided inspiration for subsequent work [66] that attempts to address specific requirements and challenges in deploying a softwaredefined cellular network.

Odin[92] introduces programmability in enterprise wireless LAN environments. In particular, it builds an access point abstraction on the controller that separates the association state from the physical access point, enabling proactive mobility management and load balancing without changes to the client.

At the other end of the spectrum, OpenRadio [23] focuses on deploying a programmable wireless data plane that provides flexibility at the PHY and MAC layers (as opposed to layer-3 SDN) while meeting strict performance and time deadlines. The system would be designed to provide a modular interface that is able to process traffic subsets using different protocols such as WiFi, WiMAX, 3GPP LTE-Advanced, etc. Based on the idea of separation of the decision and forwarding planes, an operator may express decision plane rules and corresponding actions, which are assembled from processing plane modules (e.g., FFT, Viterbi decoding, etc); the end result is a state machine that expresses a fully-functional protocol.

\section{Home and Small Business}

Several projects have examined how SDN could be used in smaller networks, such as those found in the home or small businesses. As these environments have become increasingly complex and prevalent with the widespread availability of lowcost network devices, the need for more careful network management and tighter security has correspondingly increased. Poorly secured networks may become unwitting targets or hosts for malware, while outages due to network configuration issues may cause frustration or lost business. Unfortunately, it is not practical to have a dedicated network administrator in every home and office.

Calvert et al. [31] assert that the first step in managing home networks is to know what is actually happening; as such, they proposed instrumenting the network gateway/controller to act 
as a "Home Network Data Recorder" to create logs that may be utilized for troubleshooting or other purposes.

Feamster [43] proposes that such networks should operate in a "plug in and forget" fashion, namely by outsourcing management to third-party experts, and that this could be accomplished successfully through the remote control of programmable switches and the application of distributed network monitoring and inference algorithms used to detect possible security problems.

In contrast, Mortier et al. [77] believe that users desire greater understanding and control over their networks' behavior; rather than following traditional policies, a home network may be better managed by their users who better understand the dynamics and needs of their environment. Towards this goal, they created a prototype network in which SDN is used to provide users a view into how their network is being utilized while offering a single point of control.

Mehdi et al. [72] argues that an Anomaly Detection System (ADS) implemented within a programmable home network provides a more accurate identification of malicious activity as compared to one deployed at the ISP; additionally, the implementation would be able to operate at line rate with no performance penalty, while, at the same, offloading the ISP from having to monitor these large number of networks. The ADS algorithm could operate alongside other controller services, such as a HomeOS that may react to suspicious activity and report anomalies to the ISP or local administrator.

\section{FUtURE Directions}

As SDN is more widely adopted and protocols such as OpenFlow are further defined, new solutions are proposed and new challenges arise.

\section{A. Controller and Switch Design}

SDN raises significant scalability, performance, robustness, and security challenges. Below we review a number of research efforts focusing on addressing these issues at the switch- and controller design level.

In DIFANE [112], flow entries are proactively pushed to switches in an attempt to reduce the number of requests to the controller. Devoflow [37] proposes to handle "short-lived" flows in switches and "long-lived" flows in the controller to mitigate flow setup delay and controller overhead. The work proposed in [74] advocates replacing counters on ASIC by a stream of rule-matching records and processing them in the CPU to allow efficient access to counters. FLARE [78] is a new network node model focusing on "deeply programmable networks" that provides programmability for the data plane, the control plane, as well as the interface between them. The work presented in [26] discusses important aspects in controller design including hierarchical control, data model, scalability, and extensibility.

A recent study shows that one single controller can handle up to 6.000 .000 flows/s [3]. However, for increased scalability and especially for reliability and robustness purposes, it has been recognized that the logically-centralized controller must be physically distributed. Onix [63], Kando [52], and HyperFlow [97] use this approach to achieve robust and scalable control plane. In [65], trade-offs related to control distribution, such as staleness versus optimality and application logic complexity versus robustness to inconsistency are identified and quantified. In [54], the controller placement problem is discussed in terms of the number of controllers needed and where to place them in the network. In [35] a SDN variant inspired by MPLS is proposed along with the notions of $e d g e$ controllers and fabric controllers: the former control ingress and egress switches and handle the host-network interface, while the latter handle fabric switches and the operatornetwork interface.

Although control and measurement are two important components of network management, little thought has gone into designing APIs for measurement. The work presented in [111] proposes a software-defined traffic measurement architecture, which separates the measurement data plane from the control plane.

\section{B. Software-Defined Internetworking}

The Internet has revolutionized the way we, as individuals and as a society, live, work, conduct business, socialize, get entertainment, etc. As a result, the Internet is now considered part of our society's critical infrastructure much like the power, water, and transportation grids.

Scalability- and performance requirements from increasingly complex applications have posed a variety of challenges difficult to address with the current Internet architecture. This has led the research community to examine "clean-slate" solutions [44]. As the Internet has grown beyond the point at which a "flag day", such as the one used to "upgrade" the ARPANET with the TCP/IP protocol suite, would be realistic, another considerable challenge is evolving its physical infrastructure and protocols. A notable example is the deployment of IPv6: despite over a decade in the standards track and two worldwide deployment events, IPv4 still makes up the majority of Internet traffic.

Much of the current work on SDN examines or proposes solutions within the context of a single administrative domain which matches quite well SDN's logically centralized control model. However, environments whose administration is inherently decentralized, like the Internet, call for a control plane that is logically distributed. This will allow participating autonomous systems (ASes) to be controlled independently by their own (logically centralized and possibly physically distributed) controller. To-date, a few efforts have explored the idea of a Software-Defined Internet. For example, the work in [83] proposes a software-defined Internet architecture that borrows from MPLS the distinction between network edge and core to split tasks between inter-domain and intradomain components. As only the boundary routers and their associated controller in each domain are involved in interdomain tasks, changes to inter-domain service models would be limited to software modifications at the inter-domain controllers rather than the entire infrastructure. Examples of how this architecture could be used to realize new Internet services 
such as information-centric networking, and middlebox service sharing are explored.

Another approach to inter-AS routing [24] uses NOX and OpenFlow to implement BGP-like functionality. Alternatively, an extensible session protocol [62] supports application-driven configuration of network resources across domains.

\section{Controller-Service Interaction}

While controller-switch ("southbound") interaction is fairly well defined in protocols such as OpenFlow and ForCES, there is no standard for interactions between controllers and network services or applications ("northbound"). One possible explanation is that the northbound interface is defined entirely in software, while controller-switch interactions must enable hardware implementation.

If we think of the controller as a "network operating system", then there should be a clearly defined interface by which applications can access the underlying hardware (switches), co-exist and interact with other applications, and utilize system services (e.g. topology discovery, forwarding), without requiring the application developer to know the implementation details of the controller. While there are several controllers that exist, their application interfaces are still in the early stages and independent from each other.

Some proposals (e.g., Procera [102], Frenetic [45], FML [56], Nettle [101]) advocate the use of a network configuration language to express policies. For example, Procera [102] builds a policy layer on top of existing controllers to interface with configuration files, GUIs, and external sensors; the proposed policy layer is responsible for converting highlevel policies to flow constraints given to be used by the controller. In [61], network configuration and management mechanisms are proposed that focus on enabling changes to network condition and state, supporting network configuration and policy definitions, and providing visibility and control over tasks for network diagnostics and troubleshooting. The specification of a northbound interface via a policy layer and a high level language such as Procera is discussed.

Additionally, the northbound API should allow applications to apply different policies to the same flow (e.g. forwarding by destination and monitoring by source IP). The work in [75] proposes modularization to avoid that rules installed to perform one task do not override other rules. This is accomplished by means of an abstraction layer implemented with a language based on Frenetic.

Until a clear northbound interface standard emerges, SDN applications will continue to be developed in an "ad hoc" fashion and the concept of flexible and portable "network apps" may have to wait.

\section{Virtualization and Cloud Services}

The demand for virtualization and cloud services has been growing rapidly and attracting considerable interest from industry and academia. The challenges it presents include rapid provisioning, efficient resource management, and scalability which can be addressed using SDN's control model.
For example, FlowVisor [89] and AutoSlice [28] create different slices of network resources (e.g., bandwidth, topology, $\mathrm{CPU}$, forwarding table), delegate them to different controllers, and enforce isolation between slices. Other SDN controllers can be used as a network backend to support virtualization in cloud operating systems, such as Floodlight for OpenStack [5] and NOX for Mirage [2]. FlowN [41] aims to offer a scalable solution for network virtualization by providing an efficient mapping between virtual and physical networks and by leveraging scalable database systems.

In [47], an algorithm for efficient migration with bandwidth guarantees using OpenFlow was proposed. LIME [59] is a SDN-based solution for live migration of Virtual Machines, which handles the network state during migration and automatically configures network devices at new locations. NetGraph [84] provides a set of APIs for customers to access its virtual network functions such as real-time monitoring and diagnostics.

On the context of cloud data centers providing Infrastructure as a Service (IaaS), [105] presents a management framework for resources in cloud data centers and addresses multiple management issues. In this paper, authors propose a datacentric and event-driven architecture with open management interfaces, that leverages SDN techniques to integrate network resources into datacenter orchestration and service provisioning with the aim of improving service-level agreements and faster service delivery.

\section{E. Information-Centric Networking}

Information-Centric Networking (ICN) is a new paradigm proposed for the future architecture of the Internet, which aims to increase the efficiency of content delivery and content availability. This new concept has been popularized recently by a number of architecture proposals, such as ContentCentric Networking (CCN), also known as the Named Data Networking (NDN) project [57]. Their driving motivation is that the current Internet is information-driven, yet networking technology is still focused on the idea of location-based addressing and host-to-host communication. By proposing an architecture that addresses named data rather than named hosts, content distribution is implemented directly into the network fabric rather than relying on the complicated mapping, availability, and security mechanisms currently used to map content to a single location.

The separation between information processing and forwarding in ICN is aligned with the decoupling of the data plane and control plane in SDN. The question then becomes how to combine ICN with SDN towards "Software-Defined Information-Centric Networks". A number of projects [100], [27], [91], [93], [81] have proposed using SDN concepts to realize ICNs. As OpenFlow expands to support customized header matchings, SDN can be employed as key enabling technology for ICNs.

\section{F. Heterogeneous Network Support}

Future internets will become increasingly more heterogeneous interconnecting users and applications over networks ranging from wired, infrastructure-based wireless 
(e.g., cellular-based networks, wireless mesh networks), to infrastructure-less wireless networks (e.g. mobile ad-hoc networks, vehicular networks). In the meantime, mobile traffic has been increasing exponentially over the past several years, and is expected to increase 18-fold by 2016, with more mobile-connected devices than the world's population, which is already a reality [22]. As mobile devices with multiple network interfaces become commonplace, users will demand high quality communication service regardless of location or type of network access. Self-organizing networks (e.g., wireless multi-hop ad-hoc networks) may form to extend the range of infrastructure-based networks or handle episodic connectivity disruptions. Self-organizing networks may thus enable a variety of new applications such as cloud-based services, vehicular communication, community services, healthcare delivery, emergency response, and environmental monitoring, to name a few. Efficient content delivery over wireless access networks will become essential, and self-organizing networks may become a prevalent part of the future hybrid Internet.

A major challenge facing future networks is efficient utilization of resources; this is especially the case in wireless multi-hop ad-hoc networks as the available wireless capacity is inherently limited. This is due to a number of factors including the use of shared physical medium compounded, wireless channel impairments, and the absence of managed infrastructure. Though these self-organizing networks can be used to supplement or "fill the gaps" in an overburdened infrastructure [85], their lack of dedicated resources and shifting connectivity makes capacity sharing difficult. The heterogeneous characteristics of the underlying networks (e.g., physical medium, topology, stability) and nodes (e.g., buffer size, power limitations, mobility) also add another important factor when considering routing and resource allocation.

SDN has the potential to facilitate the deployment and management of network applications and services with greater efficiency. However, SDN techniques to-date, such as OpenFlow, largely target infrastructure-based networks. They promote a centralized control mechanism that is ill-suited to the level of decentralization, disruption, and delay present in infrastructure-less environments.

While previous work has examined the use of SDN in wireless environments, the scope has primarily focused on infrastructure-based deployments (e.g., WiMAX, Wi-Fi access points). A notable example is the OpenRoads project [109], which envisioned a world in which users could freely move between wireless infrastructures while also providing support to the network provider. Other works such as [81], [36], [38] have examined OpenFlow in wireless mesh environments.

\section{CONCLUDING REMARKS}

In this paper, we provided an overview of programmable networks and, in this context, examined the emerging field of Software-Defined Networking (SDN). We look at the history of programmable networks, from early ideas until recent developments. In particular we described the SDN architecture in detail as well as the OpenFlow [71] standard. We presented current SDN implementations and testing platforms and examined network services and applications that have been developed based on the SDN paradigm. We concluded with a discussion of future directions enabled by SDN ranging from support for heterogeneous networks to Information Centric Networking (ICN).

\section{REFERENCES}

[1] Beacon. https://openflow.stanford.edu/display/Beacon/Home.

[2] Connected cloud control: Openflow in mirage. http://www.openmirage.org/blog/announcing-mirage-openflow.

[3] Controller performance comparisons. http://www.openflow.org/wk/index.php/ Controller_Performance_Comparisons.

[4] Devolved Control of ATM Networks. http://www.cl.cam.ac.uk/research/srg/netos/old-projects/dcan/\#pub.

[5] Floodlight, an open sdn controller. http://floodlight.openflowhub.org/.

[6] Helios by nec. http://www.nec.com/.

[7] Indigo: Open source openflow switches http://www.openflowhub.org/display/Indigo/.

[8] Jaxon:java-based openflow controller. http://jaxon.onuos.org/.

[9] Mul. http://sourceforge.net/p/mul/wiki/Home/.

[10] The nodeflow openflow controller. http://garyberger.net/?p=537.

[11] Node.js. http://nodejs.org/.

[12] ofsoftswitch13 - cpqd. https://github.com/CPqD/ofsoftswitch13.

[13] Open networking foundation. https://www.opennetworking.org/about.

[14] Open Networking Research Center (ONRC). http://onrc.net.

[15] Open vswitch and ovs-controller. http://openvswitch.org/.

[16] Pantou: $\quad$ Openflow $1.0 \quad$ for openwrt. http://www.openflow.org/wk/index.php/ Open-

Flow_1.0_for_OpenWRT.

[17] Pox. http://www.noxrepo.org/pox/about-pox/.

[18] Ryu. http://osrg.github.com/ryu/.

[19] Sdn troubleshooting simulator. http://ucb-sts.github.com/sts/.

[20] Simple Network Access Control (SNAC). http://www.openflow.org/wp/snac/.

[21] Trema openflow controller framework. https://github.com/trema/trema.

[22] Cisco visual networking index: Global mobile data traffic forecast update, 2011-2016. Technical report, Cisco, February 2012.

[23] M. Bansal, J. Mehlman, S. Katti, and P. Levis. Openradio: a programmable wireless dataplane. In Proceedings of the first workshop on Hot topics in software defined networks, pages 109-114. ACM, 2012.

[24] R. Bennesby, P. Fonseca, E. Mota, and A. Passito. An inter-as routing component for software-defined networks. In Network Operations and Management Symposium (NOMS), 2012 IEEE, pages 138-145, 2012.

[25] A. Bianco, R. Birke, L. Giraudo, and M. Palacin. Openflow switching: Data plane performance. In Communications (ICC), 2010 IEEE International Conference on, pages 1-5, May.

[26] R. Bifulco, R. Canonico, M. Brunner, P. Hasselmeyer, and F. Mir. A practical experience in designing an openflow controller. In Software Defined Networking (EWSDN), 2012 European Workshop on, pages 61-66, Oct.

[27] N. Blefari-Melazzi, A. Detti, G. Mazza, G. Morabito, S. Salsano, and L. Veltri. An openflow-based testbed for information centric networking. Future Network \& Mobile Summit, pages 4-6, 2012.

[28] Z. Bozakov and P. Papadimitriou. Autoslice: automated and scalable slicing for software-defined networks. In Proceedings of the 2012 ACM conference on CoNEXT student workshop, CoNEXT Student'12, pages 3-4, New York, NY, USA, 2012. ACM.

[29] M. Caesar, D. Caldwell, N. Feamster, J. Rexford, A. Shaikh, and J. van der Merwe. Design and implementation of a routing control platform. In Proceedings of the 2nd conference on Symposium on Networked Systems Design \& Implementation-Volume 2, pages 15-28. USENIX Association, 2005.

[30] Z. Cai, A. Cox, and T. Ng. Maestro: A system for scalable openflow control. Technical Report TR10-08, Rice University, December 2010.

[31] K. Calvert, W. Edwards, N. Feamster, R. Grinter, Y. Deng, and $\mathrm{X}$. Zhou. Instrumenting home networks. ACM SIGCOMM Computer Communication Review, 41(1):84-89, 2011.

[32] A. Campbell, I. Katzela, K. Miki, and J. Vicente. Open signaling for atm, internet and mobile networks (opensig'98). ACM SIGCOMM Computer Communication Review, 29(1):97-108, 1999.

[33] M. Canini, D. Venzano, P. Peresini, D. Kostic, and J. Rexford. A nice way to test openflow applications. NSDI, Apr, 2012 
[34] M. Casado, M. Freedman, J. Pettit, J. Luo, N. McKeown, and S. Shenker. Ethane: Taking control of the enterprise. ACM SIGCOMM Computer Communication Review, 37(4):1-12, 2007.

[35] M. Casado, T. Koponen, S. Shenker, and A. Tootoonchian. Fabric: a retrospective on evolving sdn. In Proceedings of the first workshop on Hot topics in software defined networks, HotSDN'12, pages 85-90, New York, NY, USA, 2012. ACM.

[36] A. Coyle and H. Nguyen. A frequency control algorithm for a mobile adhoc network. In Military Communications and Information Systems Conference (MilCIS), Canberra, Australia, November 2010.

[37] A. Curtis, J. Mogul, J. Tourrilhes, P. Yalagandula, P. Sharma, and S. Banerjee. Devoflow: Scaling flow management for high-performance networks. In ACM SIGCOMM, 2011.

[38] P. Dely, A. Kassler, and N. Bayer. Openflow for wireless mesh networks. In Proceedings of 20th International Conference on Computer Communications and Networks (ICCCN), pages 1-6. IEEE, 2011.

[39] A. Doria, F. Hellstrand, K. Sundell, and T. Worster. General Switch Management Protocol (GSMP) V3. RFC 3292 (Proposed Standard), June 2002.

[40] A. Doria, J. H. Salim, R. Haas, H. Khosravi, W. Wang, L. Dong, R. Gopal, and J. Halpern. Forwarding and Control Element Separation (ForCES) Protocol Specification. RFC 5810 (Proposed Standard), Mar. 2010

[41] D. Drutskoy, E. Keller, and J. Rexford. Scalable network virtualization in software-defined networks. Internet Computing, IEEE, PP(99):1-1.

[42] R. Enns. NETCONF Configuration Protocol. RFC 4741 (Proposed Standard), Dec. 2006. Obsoleted by RFC 6241.

[43] N. Feamster. Outsourcing home network security. In Proceedings of the 2010 ACM SIGCOMM workshop on Home networks, pages 37-42. ACM, 2010.

[44] A. Feldmann. Internet clean-slate design: what and why? SIGCOMM Comput. Commun. Rev., 37(3):59-64, July 2007.

[45] N. Foster, R. Harrison, M. J. Freedman, C. Monsanto, J. Rexford, A. Story, and D. Walker. Frenetic: a network programming language. In Proceedings of the 16th ACM SIGPLAN international conference on Functional programming, ICFP' 11, pages 279-291, New York, NY, USA, 2011. ACM.

[46] A. Gember, P. Prabhu, Z. Ghadiyali, and A. Akella. Toward softwaredefined middlebox networking. 2012.

[47] S. Ghorbani and M. Caesar. Walk the line: consistent network updates with bandwidth guarantees. In Proceedings of the first workshop on Hot topics in software defined networks, HotSDN'12, pages 67-72, New York, NY, USA, 2012. ACM.

[48] A. Greenberg, G. Hjalmtysson, D. Maltz, A. Myers, J. Rexford, G. Xie, H. Yan, J. Zhan, and H. Zhang. A clean slate 4 d approach to network control and management. ACM SIGCOMM Computer Communication Review, 35(5):41-54, 2005.

[49] N. Gude, T. Koponen, J. Pettit, B. Pfaff, M. Casado, N. McKeown, and S. Shenker. Nox: towards an operating system for networks. ACM SIGCOMM Computer Communication Review, 38(3):105-110, 2008.

[50] N. Handigol, B. Heller, V. Jeyakumar, D. Maziéres, and N. McKeown. Where is the debugger for my software-defined network? In Proceedings of the first workshop on Hot topics in software defined networks, HotSDN' 12, pages 55-60, New York, NY, USA, 2012. ACM.

[51] N. Handigol, S. Seetharaman, M. Flajslik, N. McKeown, and R. Johari. Plug-n-serve: Load-balancing web traffic using openflow. $A C M$ SIGCOMM Demo, 2009

[52] S. Hassas Yeganeh and Y. Ganjali. Kandoo: a framework for efficient and scalable offloading of control applications. In Proceedings of the first workshop on Hot topics in software defined networks, HotSDN '12, pages 19-24, New York, NY, USA, 2012. ACM.

[53] B. Heller, S. Seetharaman, P. Mahadevan, Y. Yiakoumis, P. Sharma, S. Banerjee, and N. McKeown. Elastictree: Saving energy in data center networks. In Proceedings of the 7th USENIX conference on Networked systems design and implementation, pages 17-17. USENIX Association, 2010.

[54] B. Heller, R. Sherwood, and N. McKeown. The controller placement problem. In Proceedings of the first workshop on Hot topics in software defined networks, HotSDN '12, pages 7-12, New York, NY, USA, 2012. ACM.

[55] T. Henderson, M. Lacage, G. Riley, C. Dowell, and J. Kopena. Network simulations with the ns-3 simulator. SIGCOMM demonstration, 2008.

[56] T. L. Hinrichs, N. S. Gude, M. Casado, J. C. Mitchell, and S. Shenker Practical declarative network management. In Proceedings of the 1st ACM workshop on Research on enterprise networking, WREN '09, pages 1-10, New York, NY, USA, 2009. ACM.
[57] V. Jacobson, D. Smetters, J. Thornton, M. Plass, N. Briggs, and R. Braynard. Networking named content. In Proceedings of the 5th international conference on Emerging networking experiments and technologies, pages 1-12. ACM, 2009.

[58] M. Jarschel, S. Oechsner, D. Schlosser, R. Pries, S. Goll, and P. TranGia. Modeling and performance evaluation of an openflow architecture. In Teletraffic Congress (ITC), 2011 23rd International, pages 1-7, Sept.

[59] E. Keller, S. Ghorbani, M. Caesar, and J. Rexford. Live migration of an entire network (and its hosts). In Proceedings of the 11th ACM Workshop on Hot Topics in Networks, HotNets-XI, pages 109-114, New York, NY, USA, 2012. ACM.

[60] A. Khurshid, W. Zhou, M. Caesar, and P. B. Godfrey. Veriflow: verifying network-wide invariants in real time. In Proceedings of the first workshop on Hot topics in software defined networks, HotSDN '12, pages 49-54, New York, NY, USA, 2012. ACM.

[61] H. Kim and N. Feamster. Improving network management with software defined networking. Communications Magazine, IEEE, 51(2):114-119, February.

[62] E. Kissel, G. Fernandes, M. Jaffee, M. Swany, and M. Zhang. Driving software defined networks with xsp. In SDN12: Workshop on Software Defined Networks, 2012

[63] T. Koponen, M. Casado, N. Gude, J. Stribling, L. Poutievski, M. Zhu, R. Ramanathan, Y. Iwata, H. Inoue, T. Hama, et al. Onix: A distributed control platform for large-scale production networks. OSDI, Oct, 2010.

[64] B. Lantz, B. Heller, and N. McKeown. A network in a laptop: rapid prototyping for software-defined networks. In Proceedings of the Ninth ACM SIGCOMM Workshop on Hot Topics in Networks, 2010.

[65] D. Levin, A. Wundsam, B. Heller, N. Handigol, and A. Feldmann. Logically centralized?: state distribution trade-offs in software defined networks. In Proceedings of the first workshop on Hot topics in software defined networks, HotSDN '12, pages 1-6, New York, NY, USA, 2012. ACM.

[66] L. Li, Z. Mao, and J. Rexford. Toward software-defined cellular networks. 2012

[67] T. A. Limoncelli. Openflow: a radical new idea in networking. Commun. ACM, 55(8):42-47, Aug. 2012.

[68] G. Lu, R. Miao, Y. Xiong, and C. Guo. Using cpu as a traffic coprocessing unit in commodity switches. In Proceedings of the first workshop on Hot topics in software defined networks, HotSDN '12, pages 31-36, New York, NY, USA, 2012. ACM.

[69] Y. Luo, P. Cascon, E. Murray, and J. Ortega. Accelerating openflow switching with network processors. In Proceedings of the 5th ACM/IEEE Symposium on Architectures for Networking and Communications Systems, ANCS '09, pages 70-71, New York, NY, USA, 2009. ACM.

[70] H. Mai, A. Khurshid, R. Agarwal, M. Caesar, P. B. Godfrey, and S. T King. Debugging the data plane with anteater. In Proceedings of the ACM SIGCOMM 2011 conference, SIGCOMM '11, pages 290-301, New York, NY, USA, 2011. ACM.

[71] N. McKeown, T. Anderson, H. Balakrishnan, G. Parulkar, L. Peterson, J. Rexford, S. Shenker, and J. Turner. Openflow: enabling innovation in campus networks. ACM SIGCOMM Computer Communication Review, 38(2):69-74, 2008.

[72] S. Mehdi, J. Khalid, and S. Khayam. Revisiting traffic anomaly detection using software defined networking. In Recent Advances in Intrusion Detection, pages 161-180. Springer, 2011.

[73] J. E. V. D. Merwe and I. M. Leslie. Switchlets and dynamic virtual atm networks. In Proc Integrated Network Management V, pages 355-368. Chapman and Hall, 1997.

[74] J. C. Mogul and P. Congdon. Hey, you darned counters!: get off my asic! In Proceedings of the first workshop on Hot topics in software defined networks, HotSDN '12, pages 25-30, New York, NY, USA, 2012. ACM.

[75] C. Monsanto, J. Reich, N. Foster, J. Rexford, and D. Walker. Composing software-defined networks. In Proceedings 10th USENIX Symposium on Networked Systems Design and Implementation, NSDI'13, 2013.

[76] J. Moore and S. Nettles. Towards practical programmable packets. In Proceedings of the 20th Conference on Computer Communications (INFOCOM). Citeseer, 2001.

[77] R. Mortier, T. Rodden, T. Lodge, D. McAuley, C. Rotsos, A. Moore A. Koliousis, and J. Sventek. Control and understanding: Owning your home network. In Communication Systems and Networks (COMSNETS), 2012 Fourth International Conference on, pages 1-10. IEEE, 2012.

[78] A. Nakao. Flare : Open deeply programmable network node architecture. http://netseminar.stanford.edu/10_18_12.html 
[79] M. R. Nascimento, C. E. Rothenberg, M. R. Salvador, C. N. A. Corrêa, S. C. de Lucena, and M. F. Magalhães. Virtual routers as a service: the routeflow approach leveraging software-defined networks. In Proceedings of the 6th International Conference on Future Internet Technologies, CFI '11, pages 34-37, New York, NY, USA, 2011. ACM.

[80] A. Nayak, A. Reimers, N. Feamster, and R. Clark. Resonance: Dynamic access control for enterprise networks. In Proceedings of the 1st ACM workshop on Research on enterprise networking, pages 11-18. ACM 2009.

[81] X. Nguyen. Software defined networking in wireless mesh network. Msc. thesis, INRIA, UNSA, August 2012.

[82] A. Passarella. Review: A survey on content-centric technologies for the current internet: Cdn and p2p solutions. Comput. Commun., 35(1):132, Jan. 2012.

[83] B. Raghavan, M. Casado, T. Koponen, S. Ratnasamy, A. Ghodsi, and S. Shenker. Software-defined internet architecture: decoupling architecture from infrastructure. In Proceedings of the 11th ACM Workshop on Hot Topics in Networks, HotNets-XI, pages 43-48, New York, NY, USA, 2012. ACM.

[84] R. Raghavendra, J. Lobo, and K.-W. Lee. Dynamic graph query primitives for sdn-based cloudnetwork management. In Proceedings of the first workshop on Hot topics in software defined networks, HotSDN '12, pages 97-102, New York, NY, USA, 2012. ACM.

[85] B. Rais, M. Mendonca, T. Turletti, and K. Obraczka. Towards truly heterogeneous internets: Bridging infrastructure-based and infrastructureless networks. In Communication Systems and Networks (COMSNETS), 2011 Third International Conference on, pages 1-10. IEEE, 2011.

[86] M. Reitblatt, N. Foster, J. Rexford, C. Schlesinger, and D. Walker. Abstractions for network update. In Proceedings of the ACM SIGCOMM 2012 conference on Applications, technologies, architectures, and protocols for computer communication, SIGCOMM '12, pages 323-334, New York, NY, USA, 2012. ACM.

[87] J. Rexford, A. Greenberg, G. Hjalmtysson, D. Maltz, A. Myers, G. Xie, J. Zhan, and H. Zhang. Network-wide decision making: Toward a wafer-thin control plane. In Proc. HotNets, pages 59-64. Citeseer, 2004.

[88] C. E. Rothenberg, M. R. Nascimento, M. R. Salvador, C. N. A. Corrêa, S. Cunha de Lucena, and R. Raszuk. Revisiting routing control platforms with the eyes and muscles of software-defined networking. In Proceedings of the first workshop on Hot topics in software defined networks, HotSDN '12, pages 13-18, New York, NY, USA, 2012. ACM.

[89] R. Sherwood, M. Chan, A. Covington, G. Gibb, M. Flajslik, N. Handigol, T. Huang, P. Kazemian, M. Kobayashi, J. Naous, et al. Carving research slices out of your production networks with openflow. ACM SIGCOMM Computer Communication Review, 40(1):129-130, 2010.

[90] H. Shirayanagi, H. Yamada, and K. Kono. Honeyguide: A vm migration-aware network topology for saving energy consumption in data center networks. In Computers and Communications (ISCC), 2012 IEEE Symposium on, pages 000460-000467. IEEE, 2012.

[91] J. Suh, H. Jung, T. Kwon, and Y. Choi. C-flow: Content-oriented networking over openflow. In Open Networking Summit, April 2012.

[92] L. Suresh, J. Schulz-Zander, R. Merz, A. Feldmann, and T. Vazao. Towards programmable enterprise wlans with odin. In Proceedings of the first workshop on Hot topics in software defined networks, HotSDN '12, pages 115-120, New York, NY, USA, 2012. ACM.

[93] D. Syrivelis, G. Parisis, D. Trossen, P. Flegkas, V. Sourlas, T. Korakis, and L. Tassiulas. Pursuing a software defined information-centric network. In Software Defined Networking (EWSDN), 2012 European Workshop on, pages 103-108, Oct.

[94] V. Tanyingyong, M. Hidell, and P. Sjödin. Improving pc-based openflow switching performance. In Proceedings of the 6th ACM/IEEE Symposium on Architectures for Networking and Communications Systems, ANCS '10, pages 13:1-13:2, New York, NY, USA, 2010. ACM.

[95] D. Tennenhouse, J. Smith, W. Sincoskie, D. Wetherall, and G. Minden A survey of active network research. Communications Magazine, IEEE, 35(1):80-86, 1997.

[96] D. Tennenhouse and D. Wetherall. Towards an active network architecture. In DARPA Active NEtworks Conference and Exposition, 2002. Proceedings, pages 2-15. IEEE, 2002.

[97] A. Tootoonchian and Y. Ganjali. Hyperflow: A distributed control plane for openflow. In Proceedings of the 2010 internet network management conference on Research on enterprise networking, pages 3-3. USENIX Association, 2010

[98] A. Tootoonchian, S. Gorbunov, Y. Ganjali, M. Casado, and R. Sherwood. On controller performance in software-defined networks. In
USENIX Workshop on Hot Topics in Management of Internet, Cloud, and Enterprise Networks and Services (Hot-ICE), 2012.

[99] J. Van der Merwe, S. Rooney, I. Leslie, and S. Crosby. The tempesta practical framework for network programmability. Network, IEEE, 12(3):20-28, 1998.

[100] L. Veltri, G. Morabito, S. Salsano, N. Blefari-Melazzi, and A. Detti Supporting information-centric functionality in software defined networks. IEEE ICC Workshop on Software Defined Networks, June 2012.

[101] A. Voellmy and P. Hudak. Nettle: taking the sting out of programming network routers. In Proceedings of the 13th international conference on Practical aspects of declarative languages, PADL'11, pages 235-249, Berlin, Heidelberg, 2011. Springer-Verlag.

[102] A. Voellmy, H. Kim, and N. Feamster. Procera: a language for highlevel reactive network control. In Proceedings of the first workshop on Hot topics in software defined networks, HotSDN '12, pages 43-48, New York, NY, USA, 2012. ACM.

[103] A. Voellmy and J. Wang. Scalable software defined network controllers. In Proceedings of the ACM SIGCOMM 2012 conference on Applications, technologies, architectures, and protocols for computer communication, SIGCOMM '12, pages 289-290, New York, NY, USA, 2012. ACM.

[104] R. Wang, D. Butnariu, and J. Rexford. Openflow-based server load balancing gone wild. In Workshop of HotICE, volume 11, 2011.

[105] X. Wang, Z. Liu, Y. Qi, and J. Li. Livecloud: A lucid orchestrator for cloud datacenters. In Cloud Computing Technology and Science (CloudCom), 2012 IEEE 4th International Conference on, pages 341348, 2012.

[106] Z. Wang, T. Tsou, J. Huang, X. Shi, and X. Yin. draft-wang-forcescompare-openflow-forces-01.

[107] A. Wundsam, D. Levin, S. Seetharaman, and A. Feldmann. Ofrewind: enabling record and replay troubleshooting for networks. In Proceedings of the 2011 USENIX conference on USENIX annual technical conference, USENIXATC'11, pages 29-29, Berkeley, CA, USA, 2011. USENIX Association.

[108] K. Yap, M. Kobayashi, R. Sherwood, T. Huang, M. Chan, N. Handigol, and N. McKeown. Openroads: Empowering research in mobile networks. ACM SIGCOMM Computer Communication Review, 40(1):125-126, 2010.

[109] K. Yap, R. Sherwood, M. Kobayashi, T. Huang, M. Chan, N. Handigol, N. McKeown, and G. Parulkar. Blueprint for introducing innovation into wireless mobile networks. In Proceedings of the second ACM SIGCOMM workshop on Virtualized infrastructure systems and architectures, pages 25-32. ACM, 2010.

[110] S. Yeganeh, A. Tootoonchian, and Y. Ganjali. On scalability of software-defined networking. Communications Magazine, IEEE 51(2):136-141, February.

[111] M. Yu, L. Jose, and R. Miao. Software defined traffic measurement with opensketch. In Proceedings 10th USENIX Symposium on Networked Systems Design and Implementation, NSDI'13, 2013.

[112] M. Yu, J. Rexford, M. Freedman, and J. Wang. Scalable flow-based networking with difane. In Proceedings of the ACM SIGCOMM 2010 conference on SIGCOMM, pages 351-362. ACM, 2010. 